\title{
Racional e Desenho do Registro Brasileiro para Avaliação do Stent Coronário Eluidor de Biolimus A9 Nobori ${ }^{\mathrm{TM}}$
}

\author{
Investigadores do Registro eNOBORI - Brasil ${ }^{*}$
}

\section{RESUMO}

Introdução: Diversos registros de vigilância pós-comercialização, multicêntricos, nacionais e internacionais, foram idealizados e operacionalizados no último quinquênio para aferir as taxas de segurança e eficácia tardias das diversas marcas e modelos de stents farmacológicos aprovados para uso na prática clínica. Este registro tem o objetivo de documentar os resultados de pacientes consecutivos submetidos a intervenção coronária percutânea (ICP) com o implante do stent eluidor de biolimus Nobori TM (Terumo Corporation, Tóquio, Japão), tratados em hospitais públicos e privados brasileiros, com seguimento de 30 dias, 12 meses e 24 meses. Métodos: Serão incluídos 506 pacientes, com mais de 18 anos de idade, com critérios clínicos e angiográficos para ICP com implante de stent(s) coronário(s) farmacológico(s) recomendados pelas diretrizes vigentes. Será avaliado como desfecho primário o desfecho combinado relacionado ao dispositivo, aferido aos 12 meses, definido como a ocorrência de morte cardíaca, infarto agudo do miocárdio (Q e não-Q) relacionado ao vaso tratado ou revascularização da lesão-alvo guiada por isquemia. A inclusão de pacientes foi iniciada em dezembro de 2010 nos 37 centros brasileiros participantes e o encerramento está previsto para dezembro de 2011. Conclusões: A análise dos resultados deste registro multicêntrico e de seus subgrupos pré-especificados permitirá a aferição do desempenho do stent coronário farmacológico Nobori ${ }^{\mathrm{TM}}$ na prática clínica intervencionista brasileira.

DESCRITORES: Stents farmacológicos. Trombose. Reestenose coronária. Revascularização miocárdica.

\begin{abstract}
Rationale and Design of the Brazilian Registry to Assess the Nobori ${ }^{\mathrm{TM}}$ Biolimus A9 Drug-Eluting Coronary Stent

Background: Several multicenter national and international post-marketing surveillance registries have been conceived and operationalized in the last 5 years to assess the safety and efficacy of different brands and models of drug-eluting stents approved for use in the clinical practice. This registry is aimed at documenting the results of consecutive patients undergoing percutaneous coronary intervention $(\mathrm{PCI})$ with the Nobori ${ }^{\mathrm{TM}}$ drug-eluting stent (Terumo Corporation, Tokyo, Japan), treated in public and private hospitals in Brazil, at 30 days, 12 and 24 months. Methods: Five hundred and six patients over 18 years of age will be included, with clinical and angiographic criteria for $\mathrm{PCl}$ with drug-eluting stents recommended by the current guidelines. The primary endpoint is to assess the device oriented composite endpoint, defined as cardiac death, myocardial infarction (Q-wave and non-Q-wave not clearly attributable to non-target vessel) and clinically driven target lesion revascularization at 12 months. Patient inclusion started in December 2010 in 37 Brazilian sites and completion is estimated for December 2011. Conclusions: The results of this multicenter registry and its pre-specified subgroups will allow the assessment of the performance of the Nobori ${ }^{\mathrm{TM}}$ drug-eluting stent in interventional clinical practice in Brazil.
\end{abstract}

KEY-WORDS: Drug-eluting stents. Thrombosis. Coronary restenosis. Myocardial revascularization.

\footnotetext{
* A relação dos Centros Investigadores Participantes encontra-se no Apêndice.
}

Correspondência: Luiz Alberto Mattos. Av. Jandira, 550/121 - Indianópolis - São Paulo, SP, Brasil - CEP 04080-003

E-mail: pivmattos@uol.com.br

Recebido em: 1/2/2011 • Aceito em: 5/32011 
A intervenção coronária percutânea (ICP) é um método não-cirúrgico, validado por evidência científica angariada nas últimas três décadas, para desobstrução das artérias coronárias de pacientes com estenoses significativas ( $>70 \%)$, associadas a manifestações sintomáticas e/ou demonstração objetiva de isquemia miocárdica. Os dispositivos padronizados para esse procedimento, desde 1995, são os stents coronários não-farmacológicos. ${ }^{1}$

Os stents não-farmacológicos, no entanto, cursam com taxas médias de reestenose de $25 \%$, com porcentuais que variam de $5 \%$ a $70 \%$ na dependência dos perfis clínico (maior reestenose em diabéticos) e angiográfico da lesão tratada (maior reestenose em lesões $>20$ mm, em vasos com diâmetro de referência $<2,75 \mathrm{~mm}$, e em lesões reestenóticas ou ostiais).

Os stents coronários farmacológicos compartilham a mesma plataforma metálica que os stents não-farmacológicos, porém são revestidos com polímeros duráveis ou bioabsorvíveis, que promovem a liberação controlada de fármacos diversos (sirolimus, biolimus, zotarolimus, everolimus e paclitaxel), e são essas substâncias que, dotadas de ação antiproliferativa, resultam em redução significativa (em média $70 \%$ ) da hiperplasia intimal cicatricial. ${ }^{1,2}$ Os stents farmacológicos estão disponíveis para utilização na prática clínica no Brasil desde maio de 2002, e, desde então, 16 modelos comerciais foram registrados na Agência Nacional de Vigilância Sanitária (Anvisa)., ${ }^{3,4}$ As taxas de conversão na escolha do tipo de dispositivo, de stents não-farmacológicos para stents farmacológicos, são superiores a $70 \%$ nos Estados Unidos e na Europa ${ }^{2,5}$. No Brasil, como ainda não há reembolso por parte do Sistema Único de Saúde (SUS), estima-se que essa conversão seja da ordem de $30 \%$ a $40 \%$, concentrada em pacientes tratados pela saúde suplementar. ${ }^{3,6}$

Diversos registros de vigilância pós-comercialização, multicêntricos, nacionais e internacionais, foram idealizados e operacionalizados no último quinquênio, aferindo as taxas de segurança (ocorrência de trombose da prótese) e eficácia (necessidade de novo procedimento de revascularização da lesão-alvo) tardias de diversas marcas e modelos de stents farmacológicos, autorizados para utilização na prática clínica pelos departamentos de vigilância sanitária de cada país., ${ }^{1,2,7}$

O registro do stent farmacológico Nobori ${ }^{\mathrm{TM}}$ (Terumo Corporation, Tóquio, Japão) foi idealizado para confirmar a segurança e a eficácia desse dispositivo de nova geração, que difere de outros stents por apresentar polímero biodegradável e eluição do fármaco antiproliferativo biolimus A9. Em ensaios controlados prévios, esse stent demonstrou sua não-inferioridade quando comparado ao stent eluidor de paclitaxel (Taxus ${ }^{\mathrm{TM}}$ ) e sua similaridade com o modelo eluidor de sirolimus. ${ }^{8-12}$ Seguindo as recomendações das autoridades regulatórias, este registro incluirá a análise de subgrupos específicos, com base nas características dos pacientes e das estenoses submetidas a revascularização percutânea. O Registro e-NOBORI permitirá uma análise horizontal da utilização desse modelo de stent farmacológico na prática clínica diária, fornecendo detalhada percepção de sua eficácia e segurança depois de dois anos de seguimento clínico.

\section{MÉTODOS}

Este é um registro prospectivo, de braço único, multicêntrico, no qual pacientes submetidos a ICP com implante do stent farmacológico Nobori ${ }^{\mathrm{TM}}$ serão acompanhados em 1 mês, 12 meses e 24 meses pós-procedimento.

O desfecho primário, denominado desfecho combinado relacionado ao dispositivo (DCRD), aferido aos 12 meses, é definido como a ocorrência de morte cardíaca, infarto agudo do miocárdio (IAM, Q e não-Q) relacionado ao vaso tratado ou revascularização da lesãoalvo guiada por isquemia.

Os desfechos secundários são definidos como:

- DCRD em 1 mês e 24 meses;

- desfecho combinado relacionado ao paciente, definido como morte de qualquer causa, IAM ( $Q$ e não-Q) e qualquer revascularização do vaso-alvo guiada por isquemia, em 1 mês, 12 meses e 24 meses;

- óbito e IAM em 1 mês, 12 meses e 24 meses;

- óbito e IAM pós-procedimento em 1 mês, 12 meses e 24 meses;

- trombose do stent definitiva e provável (de acordo com a definição do Academic Research Consortium) em 1 mês, 12 meses e 24 meses;

- duração da terapêutica antiplaquetária dupla;

- óbito, IAM pós-procedimento e trombose do stent durante a utilização da terapêutica antiplaquetária dupla vs. os mesmos eventos após a interrupção dessa terapêutica;

- revascularização da lesão-alvo guiada por isquemia em 1 mês, 12 meses e 24 meses;

- revascularização do vaso-alvo guiada por isquemia em 1 mês, 12 meses e 24 meses;

- taxa de revascularização total (guiada ou não por isquemia) em 1 mês, 12 meses e 24 meses;

- sucesso do dispositivo definido como lesão residual $<30 \%$ após o implante do stent Nobori ${ }^{\mathrm{TM}}$;

- sucesso do procedimento definido como lesão residual $<30 \%$, sem a ocorrência de óbito, IAM (Q e não-Q) ou revascularização da lesão-alvo durante o procedimento índice.

\section{Stent Nobori ${ }^{\mathrm{TM}}$}

O stent coronário Nobori ${ }^{\mathrm{TM}}$ é um dispositivo metálico eluidor de biolimus A9, de nova geração. Apresen- 
ta 4 componentes: plataforma em aço inoxidável 316 L, montada sobre um sistema de entrega balão-expansível (Nagare ${ }^{\mathrm{TM}}$ ), com revestimento em polímero biodegradável (ácido polilático) e o fármaco imunossupressor derivado da rapamicina (biolimus A9). O stent Nobori ${ }^{\mathrm{TM}}$ recebeu aprovação regulamentar na Europa (CE Mark), em 2008, e, desde então, mais de 50 mil pacientes foram tratados na Europa, Ásia, América do Sul e África. ${ }^{2,9}$ Esse stent farmacológico está aprovado para utilização na prática clínica brasileira desde 11 de dezembro de 2008, ${ }^{13}$ e será disponibilizado aos investigadores em 4 diâmetros $(2,5 \mathrm{~mm}, 2,75 \mathrm{~mm}, 3 \mathrm{~mm}$ e 3,5 mm) e 5 extensões $(8 \mathrm{~mm}, 14 \mathrm{~mm}, 18 \mathrm{~mm}, 24 \mathrm{~mm}$ e $28 \mathrm{~mm}$ ).

\section{Seleção de pacientes e critérios de inclusão}

Serão incluídos pacientes com mais de 18 anos de idade, elegíveis para ICP por meio de implante de stents coronários, de acordo com os diâmetros e as extensões disponíveis do dispositivo investigado, e que tiverem assinado o termo de consentimento livre e esclarecido. Consideram-se elegíveis para realização do procedimento os pacientes que preencherem as recomendações clínicas obrigatórias (farmacologia adjunta pré e pós-procedimento), angiográficas e operacionais, expostas nas diretrizes brasileiras.

Este registro tem a finalidade de capturar a prática diária dos centros brasileiros de cardiologia intervencionista e estimular os investigadores a selecionar os pacientes portadores de síndromes clínicas e achados angiográficos de maior complexidade, como IAM com supradesnivelamento do segmento ST (ICP primária), estenoses localizadas em bifurcações (incluindo o tronco de coronária esquerda não protegido por revascularização prévia) ou enxertos de veia safena, oclusões crônicas e lesões reestenóticas intrastent.

\section{Centros participantes}

Os centros participantes foram selecionados por meio de convite da Terumo Medical do Brasil, após análise de questionário prévio dedicado, idealizado para verificar a possibilidade de participação neste registro. $\mathrm{O}$ investigador principal será responsável pelo gerenciamento da pesquisa em seu centro. Foram selecionados 37 centros, representativos de todas as regiões geográficas da federação. A inclusão de pacientes será competitiva, isto é, sem um limite superior de casos recrutados para cada centro.

\section{Procedimentos operacionais}

Nos pacientes selecionados deverão ser dosados, nas 24 horas prévias ao procedimento, os marcadores séricos de necrose miocárdica (creatina quinase, creatina quinase fração $\mathrm{MB}$ ou troponina) e realizado eletrocardiograma de 12 derivações. A ICP seguirá a prática rotineira já estabelecida no que se refere a seleção da via de acesso, diâmetro de introdutores vasculares, cateteres, fios-guia e balões. A pré-dilatação é obrigató- ria, assim como a cobertura total da extensão da lesãoalvo com o stent Nobori ${ }^{\mathrm{TM}}$. Múltiplos stents sequenciais podem ser implantados, com margem mínima de sobreposição de $3 \mathrm{~mm}$. Não existe limitação para o número de vasos e lesões tratados em um mesmo procedimento. Nos casos em que uma lesão não for tratada no procedimento índice, o tratamento futuro dessa lesão, dentro do prazo de 90 dias, será considerado procedimento estagiado. Recomenda-se que os investigadores utilizem o mesmo stent (Nobori ${ }^{\mathrm{TM}}$ ) em todas as lesões tratadas em um mesmo paciente. $O$ registro sugere que os procedimentos sejam guiados por meio de angiografia coronária quantitativa. É imperativo que os investigadores arquivem originais ou cópias dos procedimentos angiográficos índice e, quando aplicáveis, tardios, para averiguações que se façam necessárias.

O início do procedimento será determinado pela introdução da bainha vascular e o final, quando o cateter-guia for retirado. A hemostasia da via de acesso fica a critério do médico operador, seja por meio de compressão manual ou mecânica seja pela utilização de seladores hemostáticos.

A conduta após o procedimento seguirá a prática clínica diária dos centros participantes, sendo obrigatória a coleta de marcadores de necrose miocárdica 18 horas a 24 horas após o final da ICP, assim como a realização de eletrocardiograma. A alta hospitalar poderá ser efetivada após avaliações clínica, laboratorial e eletrocardiográfica, seguindo as rotinas clínicas de cada centro.

\section{Farmacologia adjunta}

Os pacientes deverão receber dupla antiagregação plaquetária, em consonância com as recomendações das diretrizes vigentes ${ }^{1}$ : dose de ataque de $300 \mathrm{mg}$ de aspirina por via oral (VO) e dose de manutenção de $100 \mathrm{mg}$ VO, e dose de ataque de $300 \mathrm{mg}$ a $600 \mathrm{mg}$ de clopidogrel VO e dose de manutenção de $75 \mathrm{mg}$ VO. O clopidogrel deverá ser utilizado por 12 meses e a aspirina, indefinidamente.

A administração de heparina e/ou inibidores da glicoproteína Ilb/IIla deverá seguir as recomendações das diretrizes. ${ }^{1}$

\section{Visitas clínicas}

A coleta dos desfechos clínicos que compõem os objetivos primários e secundários deste registro poderá ser efetivada presencialmente ou por contato telefônico, sendo obrigatória aos 30 dias (com uma semana de tolerância, antes ou após a data limite), 12 meses e 24 meses (com um mês de tolerância, antes ou após a data limite) pós-procedimento. O registro não recomenda a realização de nova cinecoronariografia de rotina, isto é, sem evidência de isquemia miocárdica espontânea (retorno ou surgimento de angina) ou induzida por teste funcional, associado ou não à imagem. 
A avaliação clínica nos intervalos pré-especificados deve averiguar a presença de angina, a necessidade de hospitalização, de nova cinecoronariografia, de ICP ou de cirurgia de revascularização do miocárdio, e a ocorrência de IAM.

\section{Coleta de dados}

Todas as variáveis relacionadas à inclusão dos pacientes neste registro, sejam clínicas, angiográficas, operacionais ou de seguimento clínico tardio, deverão ser preenchidas pelo investigador em uma ficha eletrônica de coleta de dados dedicada e exclusiva do registro. Essa ficha também abordará as falhas de tratamento, como insucessos relacionados à falha do dispositivo ou demais intercorrências associadas tanto ao desfecho primário como aos desfechos secundários.

\section{Análise de subgrupos pré-especificados}

Os seguintes subgrupos de pacientes serão analisados: idosos (mais de 70 anos de idade), sexo feminino, diabéticos, insuficiência renal crônica, angina estável, síndromes coronárias agudas, vasos de fino calibre $(\leq 2,75 \mathrm{~mm})$, lesões longas ( $>24 \mathrm{~mm})$, bifurcações, pacientes com múltiplas lesões tratadas e reestenoses intrastent. ${ }^{1,2}$

\section{Análise estatística e cálculo da amostra}

As variáveis contínuas serão apresentadas como média e desvio padrão ou mediana e amplitude e as variáveis categóricas, como números e porcentagens. As variáveis categóricas serão comparadas com o teste de qui-quadrado e as variáveis contínuas, com o teste $t$ ou com o teste de Wilcoxon, de acordo com sua distribuição. As taxas de DCRD e dos desfechos secundários serão estimadas pelo método de Kaplan-Meier e as diferenças, pelo teste log-rank. Na avaliação dos preditores do DCRD, será utilizado modelo de regressão logística multivariada para ajustar as possíveis diferenças das características basais clínicas, angiográficas e do procedimento. Os fatores de risco independentes para DCRD serão expressos pela razão de risco e seu intervalo de confiança de $95 \%$.

Trata-se de ensaio de braço único, dimensionado para aferir o DCRD. Dados históricos, incluindo a geração anterior de stents farmacológicos, demonstram sobrevida livre desse desfecho entre $80 \%$ e $90 \%$. O cálculo atual considerou uma taxa livre de eventos similar para o stent Nobori ${ }^{\mathrm{TM}}$, estimando sobrevida livre de DCRD de 90\% (taxa de eventos adversos de até $10 \%)$. Utilizando-se o teste sequencial binomial, estimou-se o tamanho de amostra em 460 pacientes. Considerando a possível taxa de abandono de $10 \%$, o tamanho de amostra final foi estimado em 506 pacientes.

\section{Desvio de utilização do dispositivo}

Desvio de utilização do dispositivo é definido como o não atendimento das especificações de desempe- nho ou do uso especificado no rótulo. No caso de falha de um dispositivo, o investigador deve providenciar a devolução do dispositivo para a Terumo Medical do Brasil, acompanhado, se possível, da embalagem original que permita sua identificação (número de lote e número de série), além do preenchimento de ficha eletrônica específica. Essa comunicação deverá ser efetivada até 24 horas após a ocorrência. O investigador deverá utilizar as definições pré-definidas (Tabela) para avaliar a relação do evento adverso ao uso do dispositivo do estudo.

\section{Eventos adversos}

Os eventos clínicos adversos devem ser coletados e informados em ficha eletrônica específica. Os investigadores, além de reportar a ocorrência de desfechos clínicos relevantes, devem classificar esses eventos como: leve (sinal ou sintoma sem interferência na atividade normal do paciente ou transitório, resolvendo-se sem tratamento), moderado (interferência na atividade normal do paciente e/ou necessidade de tratamento sintomático) ou grave (sintoma ou sintomas causando grave desconforto e impacto significativo na atividade normal do paciente, com necessidade de tratamento).

\section{Financiamento}

Este registro é propriedade da Terumo Medical do Brasil, patrocinadora da pesquisa.

\section{Controle da qualidade de dados}

O instituto de pesquisa Cardiovascular Research Center Ltda. (CRC) foi selecionado para aplicação, controle e vigilância da coleta dos dados desta pesquisa. Esse instituto também auxilia e controla a submissão do protocolo desta pesquisa aos comitês de ética de cada centro participante, assim como efetiva o treinamento dos investigadores e a coleta dos dados, por meio do preenchimento da ficha eletrônica específica.

\section{Aspecto éticos}

O protocolo foi aprovado pelo Comitê de Ética em Pesquisa do Instituto Dante Pazzanese de Cardiologia (São Paulo, SP, Brasil) em 10 de agosto de 2010, e, na sequência, pelos comitês dos demais centros participantes. Os comitês são registrados no Sistema Nacional de Ética em Pesquisa (SISNEP). Todos os pacientes deverão assinar o termo de consentimento livre e esclarecido, previamente analisado e protocolado. O estudo clínico será conduzido de acordo com os princípios da Declaração de Helsinque e das Diretrizes de Boas Práticas Clínicas, em sua versão mais recente (Resolução 196/96), obedecendo às exigências legais locais e regulatórias para efetivação de pesquisa em pacientes no Brasil.

\section{Normas para publicação}

Tanto as informações como os dados gerados relacionados a este registro estarão protegidos até a primeira 
TABELA

Definições para avaliar a relação do evento adverso ao uso do dispositivo do estudo

Não relacionado

Improvável

Possível

Provável

Altamente provável
Evento definitivamente não associado à aplicação do dispositivo: evento adverso provocado por uma doença subjacente ou concomitante ou efeito de outro dispositivo ou medicamento.

Evento adverso com pouca ou nenhuma relação temporal com o dispositivo do estudo e/ou presença de etiologia alternativa mais provável.

A sequência temporal entre a aplicação do dispositivo e o

evento é tal que a relação não é improvável ou a condição do

paciente ou da terapia concomitante poderia ter causado o evento adverso.

A sequência temporal é relevante ou o evento diminui mediante conclusão da aplicação ou da remoção do dispositivo ou o evento não pode ser razoavelmente explicado pela condição do paciente.

A sequência temporal é relevante e o evento diminui mediante conclusão da aplicação ou da remoção do dispositivo ou reaparece em nova aplicação do dispositivo (reintrodução). publicação. Os investigadores concordam em utilizar essas informações para o único propósito de concluir o estudo e para nenhum outro fim sem o consentimento por escrito do patrocinador e do Comitê Operacional Executivo e Diretivo.

No final do estudo será preparado um resumo com os principais resultados, que será posteriormente apresentado em um fórum internacional apropriado. Um manuscrito revisado por especialistas também será preparado para publicação em periódico científico. As apresentações e os manuscritos serão confeccionados para cada subanálise predeterminada. Investigadores que se destacarem como líderes na inclusão de pacientes neste registro terão prioridade na divulgação de um dos subestudos pré-especificados, caso manifestem interesse e mediante aval do patrocinador e do comitê diretivo deste registro.

\section{Confidencialidade}

Dados e informações coletados durante este estudo serão considerados confidenciais pelo patrocinador. Os dados empregados na análise e no resumo deste estudo serão utilizados sob condição de anonimato, ou seja, sem referência aos nomes dos participantes do estudo. O acesso aos arquivos dos pacientes do estudo será limitado à equipe autorizada, da qual fazem parte o patrocinador, os investigadores e o instituto de pesquisa contratado (CRC). Pessoal regulamentar e autorizado poderá inspecionar e copiar todos os registros referentes ao estudo, mas serão envidados todos os esforços possíveis para excluir informações pessoais dos pacientes.

\section{Encerramento}

O patrocinador e os Comitês de Ética em Pesquisa irão monitorar o progresso do registro. Se necessário, o estudo poderá ser suspenso ou interrompido preco- cemente, caso sejam constatadas evidências de risco não razoável à população do estudo. Em caso de suspensão ou encerramento do estudo, o patrocinador enviará um relatório descrevendo as circunstâncias aos comitês e aos investigadores. Um estudo suspenso ou encerrado não pode ser reiniciado sem aprovação dos Comitês de Ética em Pesquisa após a necessária revisão.

\section{Temporalidade da coleta de dados}

O registro iniciou a coleta de dados em dezembro de 2010 e até fevereiro de 2011 foram incluídos 32 pacientes. O término da inclusão está estimado para dezembro de 2011.

\section{Organização}

Comitê Operacional Diretivo e Executivo: Drágica Paunovic, Danny Detiege, Eduardo Maruo, Adriana Victoria e Luiz Alberto Mattos

Propriedade intelectual: Terumo Medical do Brasil

Coordenação e supervisão: Terumo Medical do Brasil

Instituto de pesquisa: Cardiovascular Research Center Ltda. (CRC)

\section{CONFLITO DE INTERESSES}

Luiz Alberto Mattos é consultor da Terumo Medical do Brasil.

\section{APÊNDICE \\ Centros Investigadores Participantes:}

Hospital Mãe de Deus, Porto Alegre, RS: Alexandre Quadros - Hospital Moinhos de Vento, Porto Alegre, RS: Marco Wainstein - Hospital São Lucas da PUC, Porto Alegre, RS: Paulo Caramori - Instituto do Coração da Serra Gaúcha, Caxias do Sul, RS: Edie Mello de Oliveira - Hospital Nossa Senhora das Graças, 
Curitiba, PR: Álvaro Moura - Hospital Cruz Vermelha, Curitiba, PR: Rubens Darwich - Hospital Santa Cruz, Curitiba, PR: Rubens Darwich - Hospital Paraná CEDIPAR, Maringá, PR: Marcos Franchetti - Hospital do Coração de Londrina, Londrina, PR: Marden Tebet Hospital Santa Isabel, Blumenau, SC: Adrian Kormann Hospital Santa Marcelina, São Paulo, SP: Bruno L. Janella - Hospital Albert Einstein, São Paulo, SP: Fabio Sandoli Brito Jr - Instituto Dante Pazzanese de Cardiologia, São Paulo, SP: Fausto Feres - Hospital Bandeirantes, São Paulo, SP: Helio Castello Jr - Hospital Beneficência Portuguesa, São Paulo, SP: Jose Armando Mangione; Décio Salvadori Júnior - Hospital e Maternidade Celso Pierro, Campinas, SP: Jose Saraiva Hospital e Maternidade Madre Theodora, Campinas, SP: Pablo Teixeirense - Hospital Vera Cruz, Campinas, SP: Silvio Gioppato - Hospital Ana Costa - Endocor, Santos, SP: Leonardo Barroso - Santa Casa de Misericórdia de Marília, Marília, SP: Luiz Alberto Mattos Santa Casa Misericórdia de Santos, Santos, SP: Luiz Claudio Carvalho - Status Cor, Rio de Janeiro, RJ: Esmeralci Ferreira - Hospital Naval Marcilio Dias, Rio de Janeiro, RJ: Maria Cristina Ferreira - Rede D'Or, Rio de Janeiro, RJ: Miguel Rati - Procordis, Niterói, RJ: Bernardo Diniz - Instituto de Cardiologia do Espírito Santo, Vitória, ES: Renato Serpa - UNICOR, Linhares, ES: João Tinoco de Paula - Centro Médico Hospitalar Vila Velha, Vila Velha, ES: Paulo César Loureiro Instituto do Coração do Triângulo Mineiro, Uberlândia, MG: Roberto Botelho - Hospital Vera Cruz, Patos de Minas, MG: Alaor Mendes Jr - Hospital Vila da Serra, Belo Horizonte, MG: João Carlos Dias - Hospital Madre Teresa, Belo Horizonte, MG: Marcos Marino - Hospital Monte Sinai, Juiz de Fora, MG: Gustavo Ramalho Hospital Anchieta/Incor, Taguatinga, DF: Vicente Paulo Motta - Hospital Geral Universitário, Cuiabá, MT: Alberto Najjar - Hospital Lopes Encore, Goiânia, GO: Mauricio Prudente - Hospital Jaime da Fonte, Recife, PE: Ricardo Pontes.

\section{REFERÊNCIAS}

1. Mattos LA, Lemos Neto PA, Rassi A Jr, Marin-Neto JA, Sousa AGMR, Devito FS, et al. Diretrizes da Sociedade Brasileira de Cardiologia - Intervenção Coronária Percutânea e Métodos Adjuntos Diagnósticos em Cardiologia Intervencionista (II Edição - 2008). Arq Bras Cardiol. 2008;91(4 Supl 1):1-58.

2. European Association for Percutaneous Cardiovascular Interventions, Wijns W, Kolh P, Danchin N, Di Mario C, Falk V,
Folliguet T, et al. Guidelines on myocardial revascularization: the Task Force on Myocardial Revascularization of the European Society of Cardiology (ESC) and the European Association for Cardio-Thoracic Surgery (EACTS). Eur Heart J. 2010;31(20):2501-55.

3. Cardoso CO, Quadros AS, Mattos LA, Gottschall CA, SarmentoLeite RE, Marin-Neto JA. Perfil de uso dos stents farmacológicos no Brasil: dados da Central Nacional de Intervenções Cardiovasculares (CENIC). Arq Bras Cardiol. 2007;89(6):356-61.

4. Brasil. Ministério da Saúde. DATASUS. Informações em saúde: assistência à saúde [Internet]. [citado 2010 dez. 30]. Disponível em: http://www.datasus.gov.br

5. Kirtane AJ, Gupta A, Iyengar S, Moses JW, Leon MB, Applegate $R$, et al. Safety and efficacy of drug-eluting and bare metal stents: comprehensive meta-analysis of randomized trials and observational studies. Circulation. 2009;119(25):3198-206.

6. Polanczyk CA, Wainstein MV, Ribeiro JP. Custo-efetividade dos stents recobertos com rapamicina em procedimentos percutâneos coronarianos no Brasil. Arq Bras Cardiol. 2007;88(4): 464-74.

7. Dhruva SS, Bero LA, Redberg RF. Strength of study evidence examined by the FDA in premarket approval of cardiovascular devices. JAMA. 2009;302(24):2679-85.

8. Chevalier B, Serruys PW, Silber S, Garcia E, Suryapranata H, Hauptmann K, et al. Randomised comparison of Nobori, biolimus A9-eluting coronary stent with a Taxus(R), paclitaxeleluting coronary stent in patients with stenosis in native coronary arteries: the Nobori 1 trial. Eurolntervention. 2007; 2(4):426-34.

9. Chevalier B, Silber S, Park SJ, Garcia E, Schuler G, Suryapranata $\mathrm{H}$, et al., for NOBORI 1 Clinical Investigators. Randomized comparison of the Nobori Biolimus A9-eluting coronary stent with the Taxus Liberté paclitaxel-eluting coronary stent in patients with stenosis in native coronary arteries: the NOBORI 1 trial-Phase 2. Circ Cardiovasc Interv. 2009;2(3):188-95.

10. Ostojic M, Sagic D, Beleslin B, Jung R, Perisic Z, Jagic N, et al. First clinical comparison of Nobori-Biolimus A9 eluting stents with Cypher-Sirolimus eluting stents: Nobori Core nine months angiographic and one year clinical outcomes. EuroIntervention. 2008;3(5):574-9.

11. Lim CC, van Gaal WJ, Testa L, Cuculi F, Arnold JR, Karamitsos $\mathrm{T}$, et al. With the "universal definition", measurement of creatine kinase-myocardial band rather than troponin allows more accurate diagnosis of periprocedural necrosis and infarction after coronary intervention. J Am Coll Cardiol. 2011; 57(6):653-61.

12. Holmes DR, Kereiakers DJ, Garg S, Serruys PW, Dehmer GJ, Ellis SG, et al. Stent thrombosis. J Am Coll Cardiol. 2010; 56(17):1357-65

13. Brasil. Ministério da Saúde. Agência Nacional de Vigilância Sanitária [Internet]. [citado 2010 dez. 30]. Disponível em: http://portal.anvisa.gov.br/wps/portal/anvisa/home/ produtossaude 